\title{
Micro-Strip Sensors Based on CVD Diamond
}

\section{RD42 Collaboration}

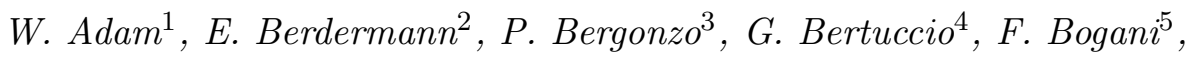
E. Borchi ${ }^{6}$, A. Brambilla ${ }^{3}$, M. Bruzzi ${ }^{6}$, C. Colledani ${ }^{7}$, J. Conway ${ }^{8}$, P. D'Angelo ${ }^{9}$, W. Dabrowski ${ }^{10}$, P. Delpierre ${ }^{11}$, A. Deneuville ${ }^{12}$,W. Dulinski ${ }^{7}$, B. van Eijk ${ }^{13}$, A. Fallou ${ }^{11}$, F. Fizzotti ${ }^{14}$, F. Foulon ${ }^{3}$, M. Friedl ${ }^{1}$, K.K. Gan ${ }^{15}$, E. Gheeraert ${ }^{12}$,

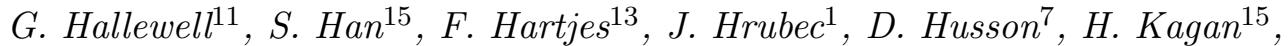
D. Kania ${ }^{15}$, J. Kaplon ${ }^{16}$, R. Kass ${ }^{15}$, T. Koeth ${ }^{8}$, M. Krammer $^{1}$, A. Logiudice ${ }^{14}$, R. Lu ${ }^{14}$, L. mac Lynne ${ }^{8}$, C. Manfredotti ${ }^{14}$, D. Meier ${ }^{16, \star}$, M. Mishina $^{18}$,

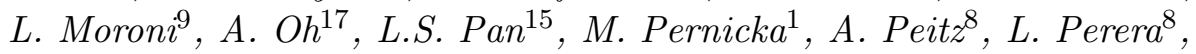

S. Pirollo ${ }^{6}$, M. Procario ${ }^{19}$, J.L. Riester ${ }^{7}$, S. Roe ${ }^{16}$, L. Rousseau ${ }^{3}$, A. Rudge ${ }^{16}$, J. Russ ${ }^{19}$, S. Sala ${ }^{9}$, M. Sampietro ${ }^{4}$, S. Schnetzer ${ }^{8}$, S. Sciortino ${ }^{6}$, H. Stelzer ${ }^{2}$,

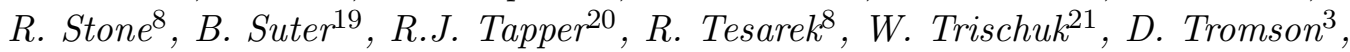

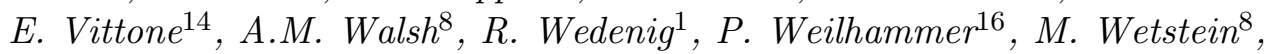
C. White ${ }^{22}$, W. Zeuner ${ }^{17}$, M. Zoeller ${ }^{15}$

1 Institut für Hochenergiephysik der Österr. Akademie d. Wissenschaften, Vienna, Austria, ${ }^{2}$ GSI, Darmstadt, Germany, ${ }^{3}$ LETI (CEA-Technologies Avancees) DEIN/SPE - CEA Saclay, Gif-Sur-Yvette, France, ${ }^{4}$ Polytechnico Milano, Italy, ${ }^{5}$ LENS, Florence, Italy, ${ }^{6}$ University of

Florence, Florence, Italy, ${ }^{7}$ LEPSI, IN2P3/CNRS-ULP, Strasbourg, France, ${ }^{8}$ Rutgers University, Piscataway, NJ, U.S.A., ${ }^{9}$ INFN, Milano, Italy, ${ }^{10}$ Faculty of Physics and Nuclear Techniques, UMM, Cracow, Poland, ${ }^{11}$ CPPM, Marseille, France, ${ }^{12}$ LEPES, Grenoble, France, ${ }^{13}$ NIKHEF, Amsterdam, Netherlands, ${ }^{14}$ Univerity of Torino, Italy, ${ }^{15}$ The Ohio State University, Columbus, OH, U.S.A., ${ }^{16}$ CERN, Geneva, Switzerland, ${ }^{17}$ II.Inst. für Exp.

Physik, Hamburg, Germany, ${ }^{18}$ FNAL, Batavia, U.S.A., ${ }^{19}$ Carnegie-Mellon University, Pittsburgh, U.S.A., ${ }^{20}$ Bristol University, Bristol, U.K., ${ }^{21}$ University of Toronto, Toronto, ON, Canada, ${ }^{22}$ Illinois Institute of Technology, Chicago, IL, U.S.A.

\section{Abstract}

In this article we present the performance of recent chemical vapour deposition (CVD) diamond micro-strip sensors in beam tests. In addition we present the first comparison of a CVD diamond micro-strip sensor before and after proton irradiation.

\footnotetext{
ऋ corresponding author: Dirk.Meier@cern.ch
} 


\section{Introduction}

Detectors in future high energy and nuclear collider experiments will be exposed to very high radiation levels [1]. There are very few materials which can withstand this level of radiation. The inherent properties of diamond indicate that it may be a radiation resistant sensor material. Recent progress in the industrial production of CVD diamond for detector applications and its fabrication into micro-strip sensors may enable it to be used for radiation resistant particle tracking close to the interaction region at future experiments $[2,3]$.

The goal of the RD42 project is the development of CVD diamond detectors for the application at high luminosity experiments at the LHC and the Tevatron. The RD42 project pursues tests of micro-strip and pixel devices based on CVD diamond using particle test beams to establish tracking performance before and after irradiation. This article presents the performance of recent CVD diamond micro-strip sensors in a particle beam and shows results from an irradiated CVD diamond strip sensor.

\section{Properties of CVD Diamond Sensors}

Fig. 1 shows a photograph of a free standing $10 \mathrm{~cm}$ diameter CVD diamond wafer to illustrate the appearance of the material as it is produced by industry [4]. The material can be grown to a thickness in a range from $400 \mu \mathrm{m}$ to several millimeter. For detector applications the material is then processed (lapped, polished, cut) to a final thickness and size depending on the application. In this paper the diamonds tested had a thickness in the range from $490 \mu \mathrm{m}$ to $520 \mu \mathrm{m}$. Fig. 2 shows an electron micrograph from the growth side of an unprocessed CVD diamond sample. The picture illustrates the poly-crystalline nature of CVD diamond. The electrical properties of CVD diamond allow charged particle detection $[5,6]$ : the energy necessary for creation of an electron-hole pair in diamond is $13 \mathrm{eV}$. This energy determines the ionization yield for a given amount of energy deposited by a particle (the mean restricted energy loss of a minimum ionizing particle mip in diamond is $\approx 145 \mathrm{keV} / 300 \mu \mathrm{m}$ and hence one mip creates $\approx 11000$ eh-pairs in $300 \mu \mathrm{m}$ diamond). The electron- and hole-mobility and lifetime determine the charge collection distance and hence the charge collected on the electrodes. In the past the chemical vapor deposition process of diamond has taken place in research reactors. The research method is now being tranfered to production reactors. For CVD diamond from production reactors the charge collection distances ranges from $150 \mu \mathrm{m}$ to $200 \mu \mathrm{m}$ corresponding to a mean charge collected of 5400 e to $7200 e$ [7]. 


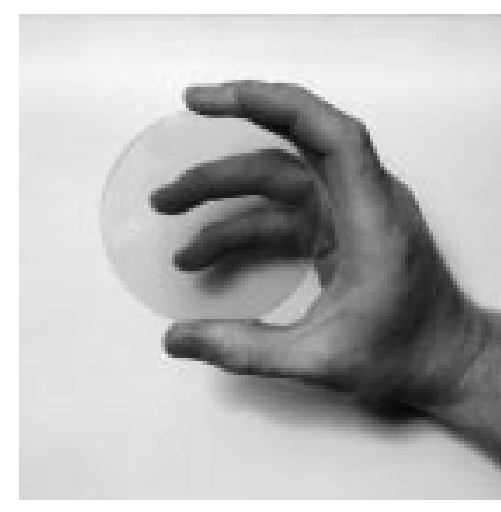

Fig. 1. Free-standing, $100 \mathrm{~mm}$ diameter CVD diamond (courtesy of De Beers Industrial Diamond Division (UK) Ltd.) [4].

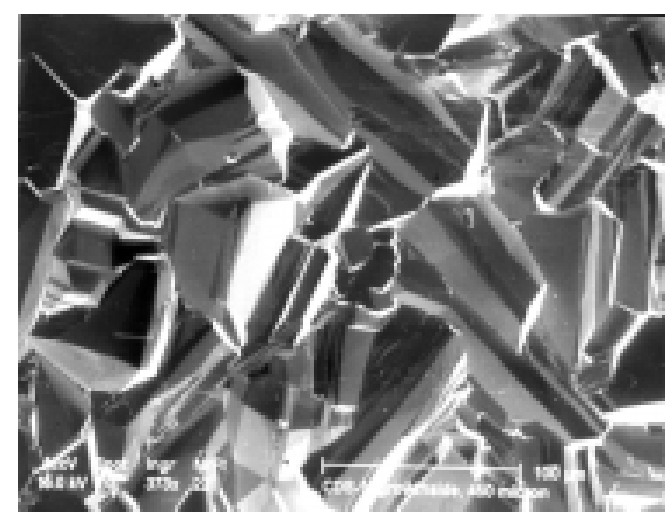

Fig. 2. Electron micrograph (SEM) from the unprocessed growth side of a CVD diamond sample.

\section{CVD Diamond Micro-Strip Sensors}

Fig. 3 shows a schematic representation of a CVD diamond micro-strip detector. For detectors described in this paper strips were deposited on the growth side and a solid electrode on the nucleation side. Charge sensitive amplifiers are directly coupled to the strips which puts the strips near (virtual) ground potential. A voltage applied to the back electrode causes an electric field inside the diamond bulk. Charged particles that traverse the bulk deposit energy and create charge carriers that move along the electric field lines. The motion of the charge carriers inside the bulk induces an electric charge on the strips (carrier velocity $\propto$ induced current). The time integral over the induced current is measured by the charge sensitive amplifiers. The position of a charged particle can be derived from the measured charge signal on the strips. Fig. 4 shows a photograph of a diamond sensor mounted on a ceramic printed circuit board. The sensors in this paper were normally biased near $1 \mathrm{~V} / \mu \mathrm{m}$ via a $100 \mathrm{M} \Omega$ resistor and a positive voltage applied to the detector backplane. A silicon reference telescope [8] was used in a $100 \mathrm{GeV} / c$ pion beam to study the performance of the sensors. The diamond sensor and its readout chips were mounted inside a light tight and electrically shielded aluminium frame inside the telescope. The analysis methods and results are also described in reference [6].

The electronic noise contribution due to the sensor depends mainly on the capacitance "seen" by the amplifier and the current into the amplifier. The capacitance "seen" by the readout amplifier is dominated by interstrip capacitance. For $25 \mu \mathrm{m}$ wide strips at $50 \mu \mathrm{m}$ pitch and $1 \mathrm{~cm}$ long strips one derives the capacitance of $\approx 1 \mathrm{pF}$ at the input of an amplifier channel. A typical current for high quality CVD diamond under normal conditions at an electric field of $1 \mathrm{~V} / \mu \mathrm{m}$ measured on circular contacts across the bulk is $14 \mathrm{pA} / \mathrm{cm}^{2}$. 


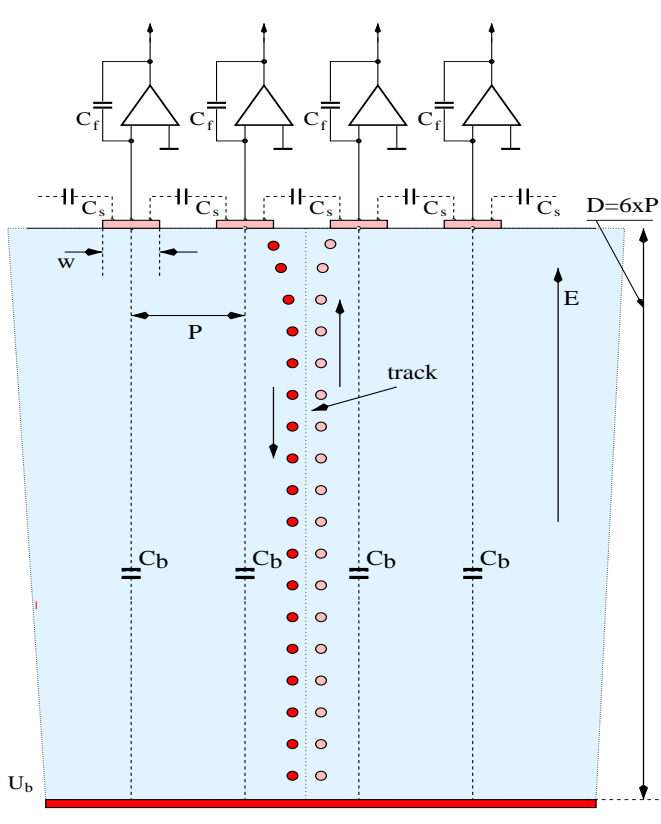

Fig. 3. Schematic representation of a diamond micro-strip sensor (side view) with electronic readout of each strip. The strips have a width, $w$, and pitch, $P$. The thickness, $D$, has been chosen to be six times the pitch in order to illustrate the relation of the pitch to thickness.

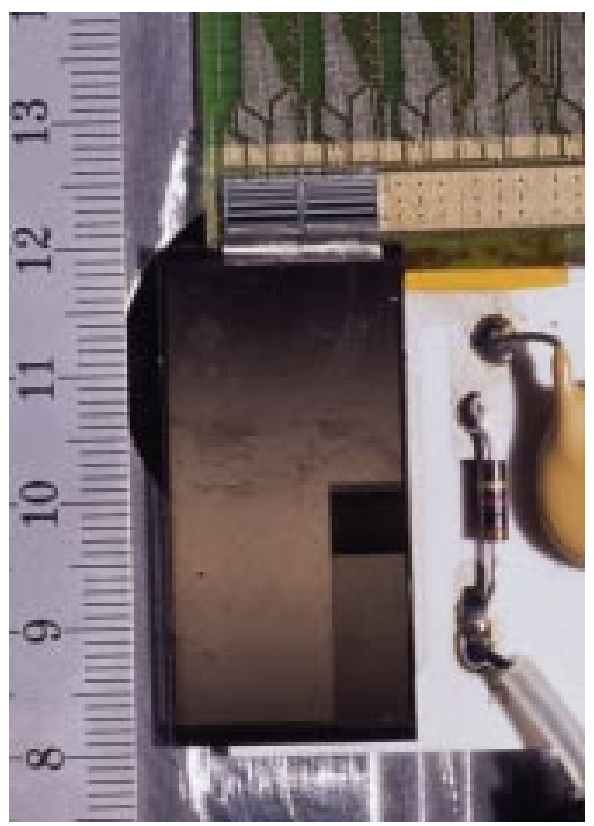

Fig. 4. Photograph of a CVD diamond micro-strip detector being read out by two VA2 chips. The strips on the diamond surface were $25 \mu \mathrm{m}$ wide with a $50 \mu \mathrm{m}$ pitch.

This implies a current of $70 \mathrm{fA}$ for $1 \mathrm{~cm}$ long strips at $50 \mu \mathrm{m}$ pitch. Using VA2 readout electronics [9] the equivalent noise charge was $\approx 100 e$ obtained on $25 \mu \mathrm{m}$ wide at $50 \mu \mathrm{m}$ pitch and $6.4 \mathrm{~mm}$ long strips.

\subsection{Charge Signals}

The response of a detector plane to a particle which intersects that plane is called hit. In a cluster analysis a hit is found by searching for a strip with the highest charge signal which exceeds a signal-to-noise threshold of 5-to-1. A hit cluster includes neighbouring strips with charge signals above a lower signalto-noise threshold of 3-to-1. The sum of charge signals in the hit cluster gives the hit cluster charge. Fig. 5 shows the distribution of 2-strip cluster charge signals in a diamond sensor, and in a silicon strip sensor of the telescope. The cluster charge is presented in units of the single strip rms noise charge. The single strip rms noise charge is $\approx 100 e \mathrm{ENC}$ in diamond (strip length $6.4 \mathrm{~mm}$ ) and $\approx 200 \mathrm{e}$ ENC in silicon (strip length $12.8 \mathrm{~mm}$ ). In the diamond sensor the most probable signal-to-noise was 50 -to-1, the mean signal-to-noise was 72-to-1. In a transparent analysis the pulse heights of the strips closest to the 
track are used independent of any threshold. The sum of charge signals closest to the track is the transparent charge signal. Fig. 6 shows the distribution of transparent charge signals in units of the single strip noise. Four distributions are shown corresponding to the transparent signal from one, two, three and four strips. The distributions shift towards higher signal as more strips are included in the sum. The most probable and mean 2-strip transparent signalto-noise is 40 -to- 1 and 58 -to- 1 . This ratio can be converted to absolute charge using the noise of $100 \mathrm{e}$. It can be seen that the start of the distribution is such that $99 \%$ of the entries in the 2-strip charge distribution are above a signal-to-noise of 10 -to-1 (above $\approx 1000 \mathrm{e}$ ).

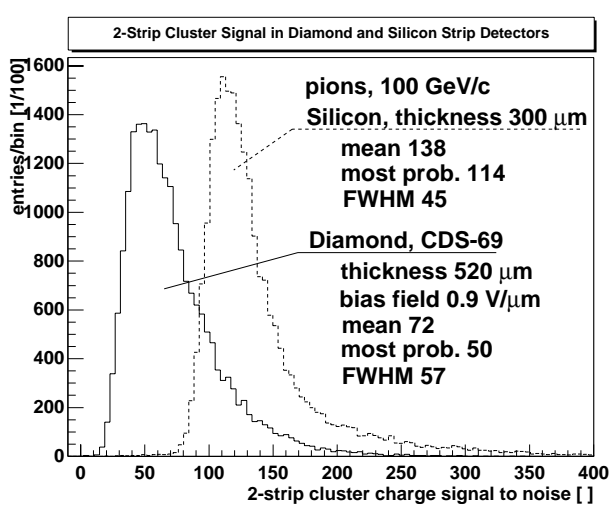

Fig. 5. Distribution of 2-strip cluster charge signals in CVD diamond (solid line) and in silicon (dashed line). The charge signal is normalized to the rms noise charge measured on single strips in diamond and in silicon respectively.

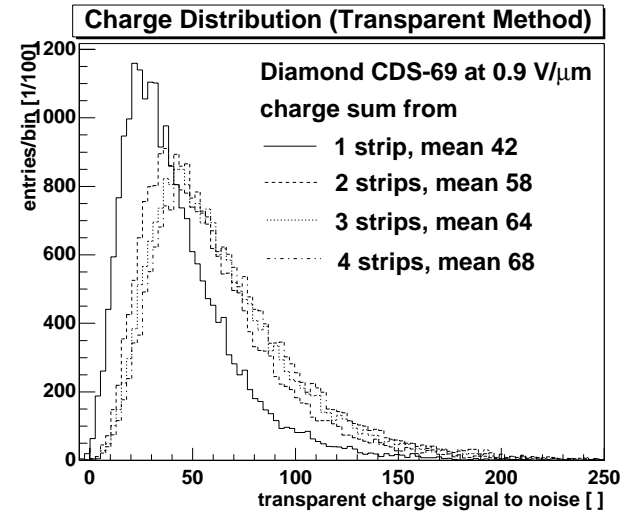

Fig. 6. Distributions of the transparent charge signals from strips nearest to the particle track. Four graphs are shown corresponding to the distribution of charges from one, two, three and four strips nearest to the track.

The transparent analysis allows one to measure the charge collected on a single strip as a function of the track position relative to the strip. Fig. 7 shows two dimensional histograms of the single strip charge signal in diamond (left) and in silicon (right). The vertical axis shows the charge signal in units of the single strip noise, the horizontal axis gives the position relative to the center of the strip which is taken to be zero (the principal strip). The entries of the histogram are grey scale coded. A profile plot is superimposed (cross marks) that gives the mean charge signal in units of the noise as a function of the track position. In the figure the central strip occupies the region from $-12.5 \mu \mathrm{m}$ to $+12.5 \mu \mathrm{m}$, while the adjacent strips occupy the region from $-37.5 \mu \mathrm{m}$ to $-62.5 \mu \mathrm{m}$ and $+37.5 \mu \mathrm{m}$ to $+62.5 \mu \mathrm{m}$. This figure illustrates that the charge signal is almost independent (flat top) of the position of the track when the track passes through the strip where the transparent mean charge signal-tonoise is 49-to-1. In between the strips a linear relation is observed indicating charge sharing between strips. Assuming uniform illumination in the interval from $-25 \mu \mathrm{m}$ to $+25 \mu \mathrm{m}$ one derives the mean charge signal-to-noise of $43-$ to-1 $(0.5 \cdot 49+0.5 \cdot 36 \approx 43)$ for single strips in agreement with the single 
strip mean value in Fig. 6. It can be seen that the transparent mean charge on adjacent neighbouring strips is around 8-to- 1 which is $15 \%$ of the level at the flat top region. The same method was used to investigate the behaviour in silicon (right histogram). The central strip in silicon is much narrower than in diamond and has a flat top with a transparent mean signal-to-noise level of 130-to-1. The adjacent readout strips are at $-50 \mu \mathrm{m}$ and $+50 \mu \mathrm{m}$. There are two intermediate strips in between of two readout strips which facilitate charge sharing. As with diamond the charge signal is almost independent of the position of the track when the track passes through the strip. In silicon the transparent mean charge on adjacent strips is around 5-to- 1 corresponding to $4 \%$ of the level at the flat top. In the case of silicon the charge observed on adjacent strips is due to charge sharing caused by intermediate strips. In diamond the charge on adjacent strips is due to the effect of carrier trapping inside the bulk.
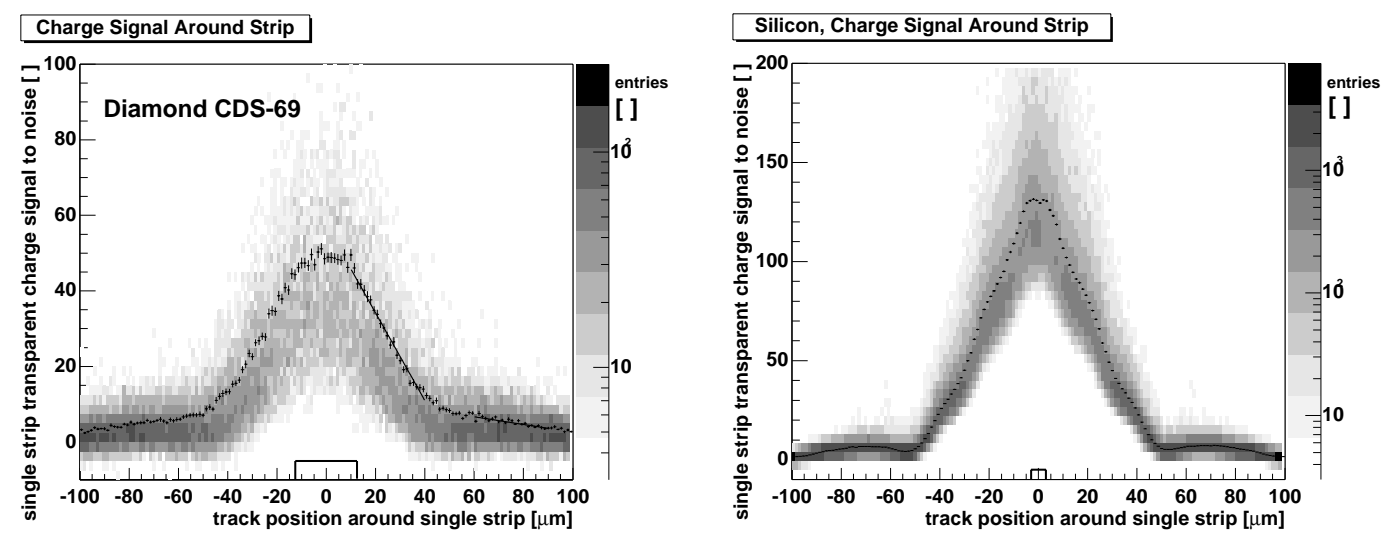

Fig. 7. Charge signal from single strips in diamond (left) and in silicon (right) as a function of the position of the track. The charge signal is normalized to the rms single strip noise charge. The histogram represents the charge distribution where the number of entries are gray scale coded; the cross marks are the mean values of the distributions parallel to the ordinate.

Fig. 8 shows two dimensional histograms of the charge signal from two strips in diamond (left) and in silicon (right). Again, the vertical axis shows the charge signal in units of the single strip noise, the horizontal axis shows the position relative to the strips. In diamond the graph of the averages (cross markers) are trapezoidal with a flat top. The flat top extends from the center of the left strip to the center of the right strip. There is nearly no charge loss between the strips. The transparent mean charge signal level of the flat top is 58-to-1 (in agreement with the 2-strip mean charge from Fig. 6). It appears that the 2-strip mean charge is the sum of the single strip charge and the charge on an adjacent strip $(49+8=57)$ as expected. In silicon the graph of the mean values (cross markers) peaks at the position of the readout strips. In the region between strips there is charge lost due to the capacitive coupling of the strips to the intermediate strips and from there to the backplane. It 
can be seen that for diamond and silicon the shape of single strip mean values from Fig. 7. determines the shape of 2-strip mean values in Fig. 8.
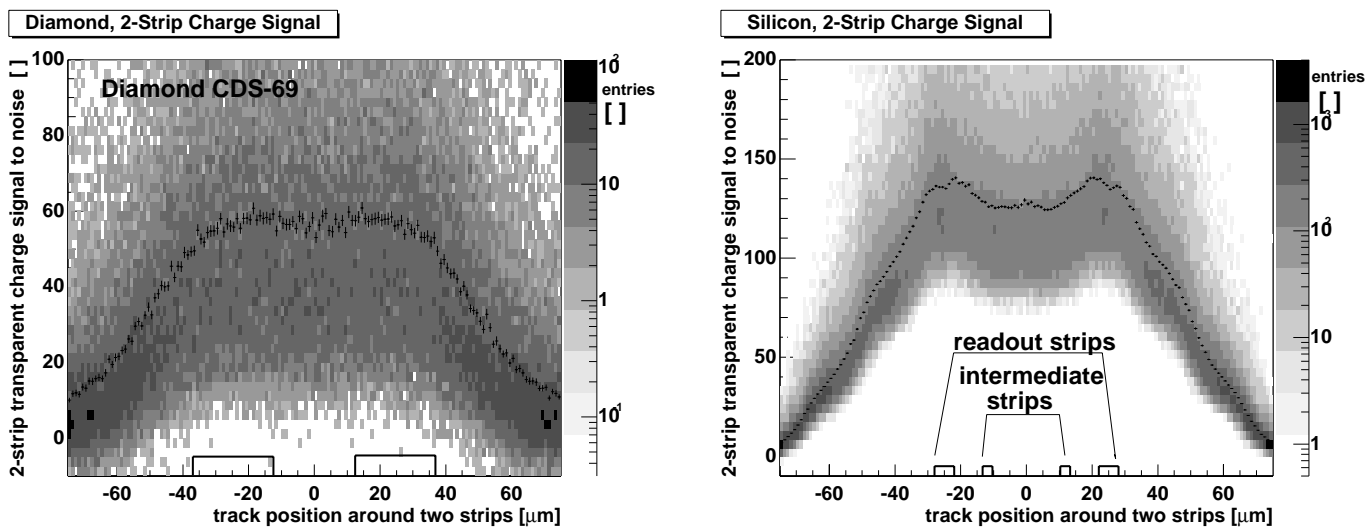

Fig. 8. Charge signal from two strips in diamond (left) and silicon (right) as a function of the position of the particle track. The charge signal is normalized to the rms single strip noise charge. The histogram represents the charge distribution where the number of entries are gray scale coded; the cross marks are the averages of the distributions parallel to the ordinate.

\subsection{Spatial Resolution}

The hit position was determined from the charge signal on strips. In particular the charge on the seed strip and the next highest charge on the adjacent strip can be used (two strip center of gravity method) to derive the position of the hit, $u_{\mathrm{h}}=u_{\mathrm{l}}+\eta_{\mathrm{r}} \cdot P$, (Eq. 1), where $u_{\mathrm{l}}$ is the position of the left strip, $P$ is the strip pitch and $\eta_{\mathrm{r}}=q_{\mathrm{r}} /\left(q_{\mathrm{r}}+q_{\mathrm{l}}\right)$ is the fractional charge on the right strip. There are other methods of determining the hit position: the digital method uses the position of the principal strip, the $K$-strip method uses the center of gravity of the charge on $K$ adjacent strips and the non-linear $\eta$-method which replaces $\eta_{\mathrm{r}}$ in Eq. 1 by a function $f$ that maps the non-uniform distribution of $\eta_{\mathrm{r}}$ into equally distributed values, $f\left(\eta_{\mathrm{r}}\right)$ [6]. Fig. 9 shows distributions of residuals using three different methods. The left measurement was obtained using a diamond tracker with $10 \mu \mathrm{m}$ wide strips on a $50 \mu \mathrm{m}$ pitch. The right measurement was obtained using the same diamond tracker with $40 \mu \mathrm{m}$ wide strips on a $50 \mu \mathrm{m}$ pitch. It can be seen that the width of the distribution (spatial resolution) depends on the method chosen. For any size strip width we find that using the two strip center of gravity method the spatial resolution is smaller than using either the digital method or the $K$-strip center of gravity method and smaller than the theoretical digital resolution of $(50 / \sqrt{12}) \mu \mathrm{m}=$ $14.4 \mu \mathrm{m}$. 

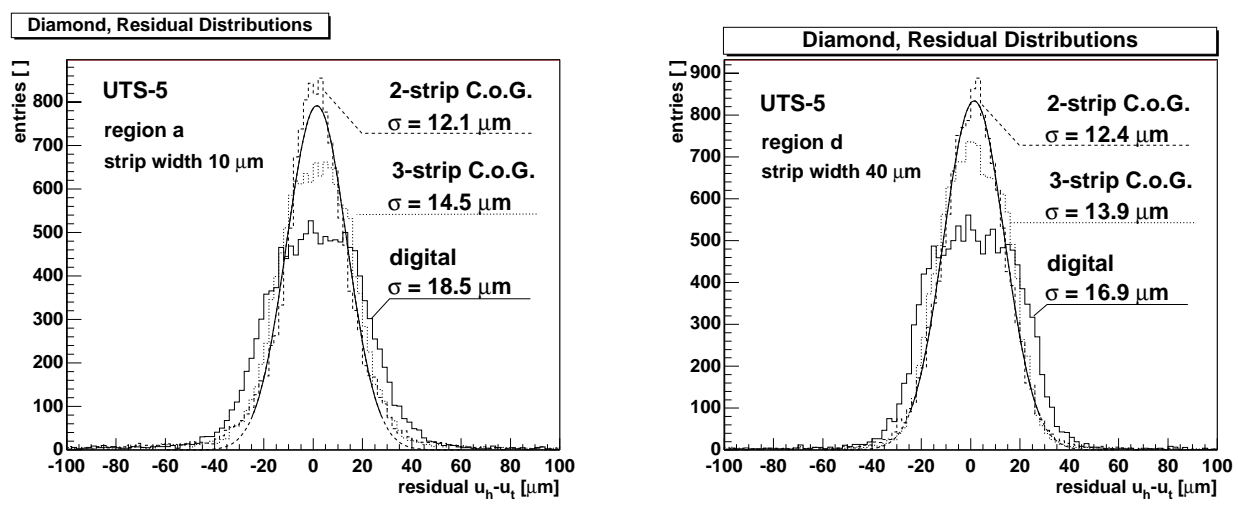

Fig. 9. Residual distributions of a CVD diamond detector with $10 \mu \mathrm{m}$ wide strips (left) and $40 \mu \mathrm{m}$ wide strips (right). The distributions for the 2-strip center of gravity, the 3 -strip center of gravity and the digital hit finding methods are shown.

\section{Performance after Proton Irradiation}

CVD diamond micro-strip sensors were irradiated at the Proton Synchrotron (PS) at CERN. The protons had a momentum of $24.3 \mathrm{GeV} / c$. The average proton flux was $4 \times 10^{10} \mathrm{p} / \mathrm{cm}^{2} / \mathrm{s}$. The absolute proton fluence was measured using aluminium foils. Fig. 10 (left) shows the transparent 2-strip signal-to-noise distribution measured in a pion test beam before and after $1 \times 10^{15} \mathrm{p} / \mathrm{cm}^{2}$. After irradiation the shape of the distribution is narrower than before irradiation and entries in the tail of the distribution appear closer to the most probable signal. The low end of the pulse height distribution changes very little. The full width at half the maximum (FWHM) is 41 after irradiation compared to 54 before irradiation. The most probable signal-to-noise was 41-to- 1 before irradiation and 35-to-1 after irradiation. Therefore we find a reduction of $15 \%$ in the most probable signal-to-noise after irradiation with $1 \times 10^{15} \mathrm{p} / \mathrm{cm}^{2}$.

Fig. 10 (right) shows the residual distribution before and after $1 \times 10^{15} \mathrm{p} / \mathrm{cm}^{2}$. It can be seen that the spatial resolution was $11.6 \mu \mathrm{m}$ before irradiation and $9.4 \mu \mathrm{m}$ after irradiation. Hence one finds that the spatial resolution improves by $18 \%$ after irradiation. This effect is statistically significant since the device was physically the same before and after irradiation and the same tracking program was used to analyze each data set.

\section{Summary and Discussion}

Results from beam tests of CVD diamond micro-strip detectors have been presented. The cluster analysis method finds a typical 2-strip most probable signal-to-noise of 50-to-1 and a mean signal-to-noise ratio of 72-to-1 in dia- 

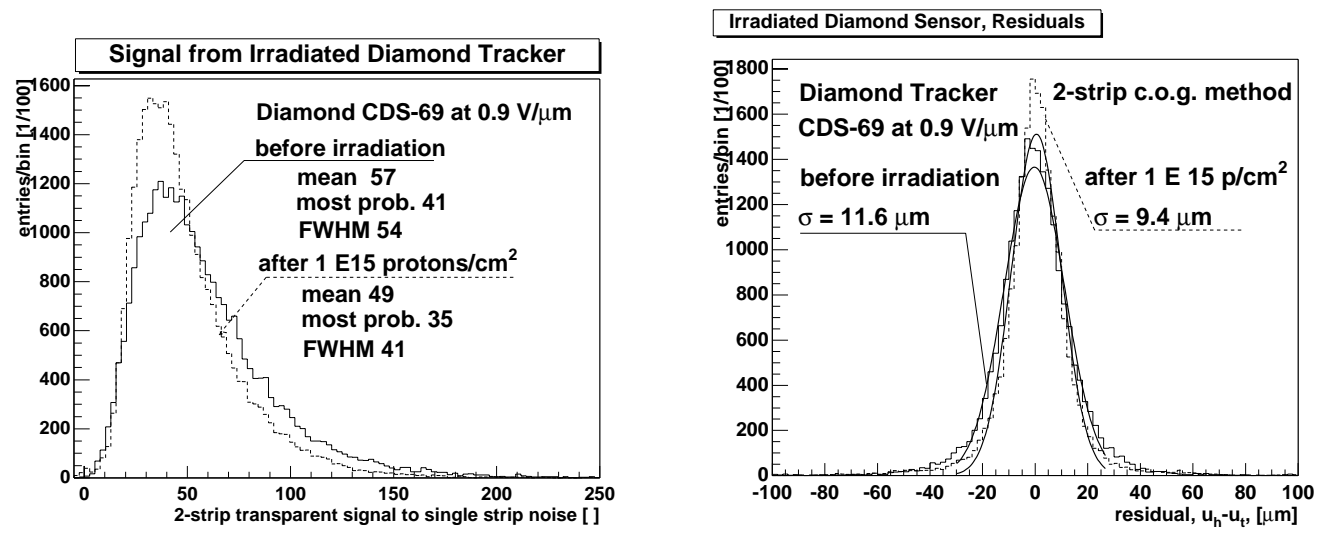

Fig. 10. Charge signal distribution (left) and distribution of residuals (right) before (solid line) and after (dashed line) irradiation with $1 \times 10^{15} \mathrm{p} / \mathrm{cm}^{2}$. The residual distributions are fitted by Gaussian functions, centered at zero, with a standard deviation, $\sigma$, which gives the spatial resolution.

mond - a result that reflects the progress made in the production of CVD diamond for detector applications. The noise charge of $\approx 100 e$ ENC in microstrip sensors is a realistic estimator for the noise in diamond pixel sensors with bump-bonded readout. For such application it is important to obtain signal separation from zero exceeding the signal threshold of pixel devices. At present signal separation various among samples but generally allows for an efficiency of $99 \%$ for thresholds below $1000 \mathrm{e}$. It should also be considered that noise in LHC type electronics for strip sensors is higher due to a much shorter shaping time: CVD diamond sensors operated with analogue SCT/DMILL readout SCT128HC demonstrated 7.2-to-1 transparent most probable signalto-noise and 10-to-1 transparent mean signal-to-noise [7]. At present highest tracking precision in CVD diamond micro-strip sensors is obtained using the 2-strip center of gravity method. The spatial resolution was always found to be better than digital $(14.4 \mu \mathrm{m})$.

Results from the first proton irradiated CVD micro-strip sensors demonstrate successful operation after $10^{15} \mathrm{p} / \mathrm{cm}^{2}$. This is a very exciting result since it encourages the development of CVD diamond pixel detectors for applications in high luminosity experiments.

\section{Acknowledgements}

We would like to thank M. Glaser et al. at CERN for irradiations and O. Runolfsson and A. Honma at CERN for excellent wire bonding. Finally we thank the organizers of INSTR'99 for this very interesting conference. 


\section{References}

[1] I. Dawson (ATLAS/SCT) et al. "The Radiation Environment in the ATLAS Inner Detector". In Proc. "th Int.Conf. on Instr. for Coll. Beam Physics. Hamamatsu, Japan, (1999).

[2] F. Borchelt et al. (RD42-Collaboration). "First Measurements with a Diamond Microstrip Detector". Nucl. Instr. Meth., A354 (1995).

[3] W. Adam et al. "First Bump-Bonded Pixel Detectors on CVD Diamond". Nucl. Instr. Meth., A436 (1999) 326-335.

[4] De Beers Industrial Diamond Division (UK) Ltd. Charters, Sunninghill, Ascot, Berkshire, SL5 9PX England.

[5] S. Zhao. "Characterization of the Electrical Properties of Polycrystalline Diamond Films". PhD thesis, Ohio State University, (1994).

[6] D. Meier. "CVD Diamond Sensors for Particle Detection and Tracking". $\mathrm{PhD}$ thesis, University of Heidelberg and CERN, atlasinfo.cern.ch/Atlas/ documentation/thesis/thesis.html, (1999).

[7] W. Adam et al. (RD42-Collaboration). "Development of Diamond Tracking Detectors for High Luminosity Experiments at the LHC". Status Report/RD42, CERN/LHCC 2000-011, (2000).

[8] C. Colledani et al. "A Submicron Precision Silicon Telescope for Beam Test Purposes". Nucl. Instr. Meth., A372 (1997) 3.

[9] Integrated Detector and Electronics (IDE) AS. "The VA Circuits". www.ideas.no. Pb.315, Veritasveien 9, N-1322 Hovik, Norway. 